period the supply of breast milk tends to diminish, both in total quantity and in relation to the needs of the growing child, and cannot be replaced by protein-rich foods such as cow's milk, which is not available. Instead the child is given pappy preparations based on cereals or starchy roots and fruits, and these usually in rather small amounts. As a result it enters a dangerous period in which growth is retarded and nealth impaired by lack of protein and calories. In the majority of tropical and sub-tropical countries deficiency states due to lack of protein and calories occur most commonly in children between 1 and 3 to 4 years. This is true of kwashiorkor, usually regarded as a manifestation of extreme protein deficiency, which produces a characteristic and well-defined clinical picture.

For these reasons, it has been suggested that the death rate in the age group 1 to 4 may provide useful evidence of a community's state of nutrition. This idea-generally familiar to nutritionists-was discussed a few years ago by V. G. Wills and J. C. Waterlow ${ }^{1}$ and recently by K. H. Uttley. ${ }^{2}$ Analysing the relevant statistics of Antigua in the West Indies, Uttley draws attention to the fact that the death rate of toddlers in poor countries is many times higher than in prosperous countries, and has not fallen in the former to nearly the same extent as the infant mortality rate. Recorded causes of the deaths of toddlers in Antigua and elsewhere do not throw much light on the contribution of under-nutrition and malnutrition to this high mortality. In Antigua "gastro-enteritis," "bronchopneumonia," and "ill-defined diseases" head the list, with "avitaminosis and other deficiency states" coming well behind. Uttley, however, comments that "gastroenteritis and bronchopneumonia ... are normally terminal events, hiding from the physician the underlying state of malnutrition which greatly facilitates their lethal attack on the child, and which is much harder to detect in their presence."

The young child in the tropics and subtropics is badly fed at a time of life when he is particularly liable to infectious disease. In practice it is often difficult to disentangle the malnutrition from the infection; each accentuates the ill-effects of the other. For example, D. Morley and his colleagues $^{3}$ have recently described the close interrelationships between measles and malnutrition in Nigeria. Further study of the factors underlying the tragic mortality in young children in many parts of the world is, however, essential. Its existence and causes have an important bearing on the future development of child health services.

\section{OESOPHAGEAL VARICES}

Oesophageal varices resulting from portal hypertension are a serious complication of hepatic cirrhosis. The tendency of these dilated vessels to bleed and thus to impair further the already reduced function of the liver leads to the wellknown features of liver-cell failure-namely, jaundice, fluid retention, and hepatic encephalopathy. This sequence often ends in death. The past few years have seen considerable changes in the practical management of patients with these complications, such as the administration by mouth of non-absorbable antibiotics and the more effective treatment of ascites. But has the prognosis improved? A. J. Garceau and T. C. Chalmers ${ }^{1}$ collected data on the duration of survival of 471 patients with cirrhosis of the liver and unequivocal evidence of oesophageal varices. The patients were seen over a two-year period by the authors and fourteen other members of the Boston Inter-Hospital Liver Group. It is significant that over $80 \%$ of these patients were alcoholics, only $8.3 \%$ had a previous history suggesting viral hepatitis, and $61 \%$ (288) of them had not bled when the varices were first diagnosed.

At the end of a year after diagnosis only one-third of the group were still alive, death usually occurring from haemorrhage in the alimentary tract or liver-cell failure. In the group of 288 patients with no previous alimentary bleeding the results were little better, $42 \%$ surviving one year. Of the total group only $5 \%$ survived five years.

The authors point out that the high mortality in their patients one year after the diagnosis of varices had been made is comparable to that of acute lymphatic leukaemia. Not all series of American cirrhotics seem to fare so badly. One-year survival figures of $65 \%$ and $67 \%$ have been recorded. ${ }^{2} 3$ It is likely that the better nutritional condition, younger age, and less extensive and less frequent occurrence of varices in these two groups account for the more favourable prognosis. What then is the reason for the gloomy Boston figures? Continuing alcoholism seems the most likely reason, for though the alcoholic with liver disease may show considerable clinical and biochemical evidence of improvement when he stops drinking (the disorder was three times commoner in males than in females in this series) continuing alcoholism leads to progressive liver-cell failure. In fact the features of livercell failure were found in $96 \%$ of the group studied-and ascites alone in over $80 \%$. The second reason for the poor prognosis follows from the high incidence of liver-cell failure-namely, that few if any patients were suitable for operative treatment, which could at least prevent further haemorrhage from varices.

Does the British cirrhotic with varices do badly? In patients with ascites and hepatic coma the prognosis is often poor and like the American patients the course may be steadily downhill. But since cirrhosis in Great Britain is not usually due to alcoholism a considerable proportion of patients with cirrhosis and varices show little evidence of liver-cell failure: their main symptoms come from the mechanical effects of portal hypertension, in particular bleeding from varices. In such a group it is impossible to say how long varices have been present before bleeding showed them up. Certainly the prognosis for these patients would not be as dismal as for those in the American series.

Perhaps the comparatively low incidence of alcoholic cirrhosis in Britain does allow shunt operations to be carried out in a greater percentage of our patients with cirrhosis of the liver and varices. Patients fit enough and specially selected for shunt surgery do well, as the figures of W. V. McDermott and his colleagues ${ }^{4}$ in the U.S.A. and of Milnes Walker and his colleagues ${ }^{5}$ in Great Britain indicate.

\footnotetext{
1 Garceau, A. J., Chalmers, T. C., and the Boston Inter-Hospital Liver Group New Engl. J. Med.. 1963, 268, 469 2 Ratnoff, O. D., and Patek, A. J., Medicine, 1942, 21, 207.

$\&$ Baker, L. A., Smith, C., and Lieberman, G., Amer. J. Med., 1959, 26, 228 McDermott, W. V., Jalazzi, H., Nardi, G. L., and Mondet, A., 1961, New Engl. J. Med., 264, 419 .

5 Walker, R. M., Shaldon, C., and Vowles, K. D. J., Lancet, 1961, 2, 727.
} 